\title{
Input-to-state dynamical stability and its Lyapunov function characterization
}

\author{
Lars Grüne, Fachbereich Mathematik, J.W. Goethe-Universität, Postfach 111932
}

60054 Frankfurt a.M., Germany, gruene@math.uni-frankfurt.de

\begin{abstract}
We present a new variant of the input-to-state stability (ISS) property which is based on using a one-dimensional dynamical system for building the class $\mathcal{K} \mathcal{L}$ function for the decay estimate and for describing the influence of the perturbation. We show the relation to the original ISS formulation and describe characterizations by means of suitable Lyapunov functions. As applications, we derive quantitative results on stability margins for nonlinear systems and a quantitative version of a small gain theorem for nonlinear systems.
\end{abstract}

Keywords: Input-to-state dynamical stability, Lyapunov function, nonlinear stability margin, small gain theorem

\section{Introduction}

The input-to-state stability (ISS) property introduced by Sontag [13] has by now become one of the central properties in the study of stability of perturbed nonlinear systems. It assumes that each trajectory $\varphi$ of a perturbed system satisfies the inequality

$$
\|\varphi(t, x, u)\| \leq\left\{\beta(\|x\|, t), \rho\left(\|u\|_{\infty}\right)\right\}
$$

for suitable functions $\beta$ of class $\mathcal{K} \mathcal{L}$ and $\rho$ of class $\mathcal{K}_{\infty}$.

While ISS has turned out to be a very useful qualitative property with many applications (see, e.g., $[1,3,6,7,8,10,12,18]$ ) and lots of interesting features (see, e.g., $[5,14,17]$ and in particular the recent survey [16]), there are some drawbacks of this property when quantitative statements are of interest. The main problem with ISS in this context is that it does not yield explicit information about what happens for vanishing perturbations, i.e., for perturbations $u$ with $u(t) \rightarrow 0$ as $t \rightarrow \infty$. Implicitly, ISS ensures that if $u(t)$ tends to 0 as $t$ tends to infinity then also $\varphi(t, x, u)$ converges to 0 for $t$ tending to infinity, but no explicit rate of convergence can be deduced. The main idea in order to overcome this difficulty is by introducing a certain "memory fading" effect into the $u$-term of the ISS formulation, an idea which was used before by Praly and Wang [10] in their notion of exp-ISS. There the perturbation is first fed into a one-dimensional control system whose output then enters the right hand side of the ISS estimate. Here, instead, we use the value of the perturbation at each time instant as an initial value of a one-dimensional dynamical system, which leads to the concept of input-to-state dynamical stability (ISDS). Proceeding this way, we are in particular able to "synchronize" the effects of past disturbances and large initial values by using the same dynamical system for both terms. It turns out that ISDS is qualitatively equivalent to ISS and, in addition, that we can pass from ISS to ISDS with only slightly larger robustness gains. 
One of the most important features of the ISS property is that it can be characterized by a dissipation inequality using a so called ISS Lyapunov function, see [17]. One of the central parts of the present paper is devoted to the construction of an ISDS Lyapunov function, which not only characterizes ISDS as a qualitative property (the qualitative equivalence ISS $\Leftrightarrow$ ISDS immediately implies that the well known ISS Lyapunov function would be sufficient for this) but also represents the respective decay rate, the overshoot gain and the robustness gain. The respective results are given in Section 3 .

We believe that there are many applications where quantitative robust stability properties are of interest. A particular area of applications are numerical investigations, where one interprets a numerical approximation as a perturbation of the original system and vice versa. We refer to the monograph [4] for results in this direction. Here we show two control theoretic applications of the ISDS property in Section 4, which also illustrate the difference to the ISS property.

\section{Input-to-state dynamical stability}

We consider nonlinear systems of the form

$$
\dot{x}(t)=f(x(t), u(t)),
$$

where we assume that $f: \mathbb{R}^{n} \times \mathbb{R}^{m} \rightarrow \mathbb{R}^{n}$ is continuous and that for each two compact subsets $K \subset \mathbb{R}^{n}$ and $W \subset \mathbb{R}^{m}$ there exists a constant $L=L(K, W)$ such that $\| f(x, u)-$ $f(y, u)\|\leq L\| x-y \|$ for all $x, y \in K$ and all $u \in W$. The perturbation functions $u$ are supposed to lie in the space $\mathcal{U}$ of measurable and locally essentially bounded functions with values in $U$, where $U$ is an arbitrary subset of $\mathbb{R}^{m}$. The trajectories of (2.1) with initial value $x$ at time $t=0$ are denoted by $\varphi(t, x, u)$.

We recall that a continuous function $\alpha: \mathbb{R}_{0}^{+} \rightarrow \mathbb{R}_{0}^{+}$is called of class $\mathcal{K}$ if it is strictly increasing with $\alpha(0)=0$, and is called of class $\mathcal{K}_{\infty}$ if, in addition, it is unbounded. A continuous function $\beta: \mathbb{R}_{0}^{+} \times \mathbb{R}_{0}^{+} \rightarrow \mathbb{R}_{0}^{+}$is called of class $\mathcal{K} \mathcal{L}$ if it is of class $\mathcal{K}_{\infty}$ in the first and strictly decreasing to 0 in the second argument. We define a continuous function $\mu: \mathbb{R}_{0}^{+} \times \mathbb{R} \rightarrow \mathbb{R}_{0}^{+}$to be of class $\mathcal{K} \mathcal{L} \mathcal{D}$ if its restriction to $\mathbb{R}_{0}^{+} \times \mathbb{R}_{0}^{+}$is of class $\mathcal{K} \mathcal{L}$ and, in addition, it is a one dimensional dynamical system, i.e., it satisfies

$$
\mu(r, t+s)=\mu(\mu(r, t), s) \text { for all } t, s \in \mathbb{R} .
$$

Observe that this condition implies $\mu(r, 0)=r$.

The expression $\|\cdot\|$ denotes the usual euclidean norm, $\|u\|_{\infty}$ is the $L_{\infty}$ norm of $u \in \mathcal{U}$ and for $t>0$ and any measurable function $g: \mathbb{R} \rightarrow \mathbb{R}_{0}^{+}$the expression $\operatorname{ess}_{\sup _{\tau \in[0, t]} g(\tau)}$ denotes the essential supremum of $g$ on $[0, t]$.

Using these notations we can now formulate the concept of input-to-state dynamical stability.

Definition 2.1 A system (2.1) is called input-to-state dynamically stable (ISDS), if there exists a function $\mu$ of class $\mathcal{K} \mathcal{L D}$ and functions $\sigma$ and $\gamma$ of class $\mathcal{K}_{\infty}$ such that the inequality

$$
\|\varphi(t, x, u)\| \leq \max \{\mu(\sigma(\|x\|), t), \nu(u, t)\} .
$$

holds for all $t \geq 0, x \in \mathbb{R}^{n}$ and all $u \in \mathcal{U}$, where $\nu$ is defined by

$$
\nu(u, t):=\operatorname{ess}_{\sup _{\tau \in[0, t]}} \mu(\gamma(\|u(\tau)\|), t-\tau)
$$


Here we call the function $\mu$ the decay rate, the function $\sigma$ the overshoot gain and the function $\gamma$ the robustness gain.

Since $\mu(\sigma(r), t)$ is of class $\mathcal{K} \mathcal{L}$, ISDS implies ISS with $\beta(r, t):=\mu(\sigma(r), t)$ and robustness gain $\rho=\gamma$.

Conversely, a straightforward application of [15, Proposition 7] shows that any class $\mathcal{K} \mathcal{L}$ function can be bounded from above by the composition of a class $\mathcal{K} \mathcal{L D}$ and a class $\mathcal{K}_{\infty}$ function, see [4, Lemma B.1.4]. Hence the only real difference between ISS and ISDS is the decay property of the $\nu(u, t)$-term. The following theorem shows how one can pass from the ISS to the ISDS formulation. For the proof see [4, Proposition 3.4.4].

Theorem 2.2 Assume that the system (2.1) is ISS for some $\beta$ of class $\mathcal{K} \mathcal{L}$ and $\rho$ of class $\mathcal{K}_{\infty}$. Then for any class $\mathcal{K}_{\infty}$ function $\gamma$ with $\gamma(r)>\rho(r)$ for all $r>0$ there exists a class $\mathcal{K} \mathcal{L D}$ function $\mu$ such that the system is ISDS with attraction rate $\mu$, overshoot gain $\sigma(r)=\beta(r, 0)$ and robustness gain $\gamma$.

For some results in this paper we will need the following assumption.

Assumption 2.3 The functions $\mu, \sigma$ and $\gamma$ in Definition 2.1 are $C^{\infty}$ on $\mathbb{R}^{+} \times \mathbb{R}$ or $\mathbb{R}^{+}$, respectively, and the function $\mu$ solves the ordinary differential equation

$$
\frac{d}{d t} \mu(r, t)=-g(\mu(r, t))
$$

for some Lipschitz continuous function $g: \mathbb{R}^{+} \rightarrow \mathbb{R}^{+}$, all $r>0$ and all $t \in \mathbb{R}$.

It was shown in [4, Appendix A] that for given nonsmooth rates and gains from Definition 2.1 one can find rates and gains arbitrarily close to the original ones, such that Assumption 2.3 holds and Definition 2.1 remains valid. Hence Assumption 2.3 is only a mild regularity condition.

\section{Lyapunov function characterization}

One of the main tools for working with ISS systems is the ISS Lyapunov function whose existence is a necessary and sufficient condition for the ISS property, see [17]. In this section we provide two theorems on a Lyapunov function characterization of the ISDS property. We start with a version for discontinuous Lyapunov functions, which can exactly represent the rate and gains in the ISDS formulation. The proof of the following theorem is given in Section 5 .

Theorem 3.1 A system (2.1) is ISDS with rate $\mu$ of class $\mathcal{K} \mathcal{L D}$ and gains $\sigma$ and $\gamma$ of class $\mathcal{K}_{\infty}$ if and only if there exists a (possibly discontinuous) ISDS Lyapunov function $V: \mathbb{R}^{n} \rightarrow \mathbb{R}_{0}^{+}$satisfying

$$
\|x\| \leq V(x) \leq \sigma(\|x\|)
$$

and

$$
V(\varphi(t, x, u)) \leq \max \{\mu(V(x), t), \nu(u, t)\}
$$

for all $x \in \mathbb{R}^{n}, t \geq 0$ and all $u \in \mathcal{U}$, where $\nu$ is given by (2.2). 
For many applications it might be desirable to have ISDS Lyapunov functions with some more regularity. The next theorem, which is also proved in Section 5, shows that if we slightly relax the sharp representation of the gains, then we can always find smooth (i.e., $\left.C^{\infty}\right)$ Lyapunov functions, at least away from the origin.

Theorem 3.2 A system (2.1) is ISDS with rate $\mu$ of class $\mathcal{K} \mathcal{L} D$ and gains $\sigma$ and $\gamma$ of class $\mathcal{K}_{\infty}$ satisfying Assumption 2.3 if and only if for each $\varepsilon>0$ there exists a continuous function $V: \mathbb{R}^{n} \rightarrow \mathbb{R}_{0}^{+}$which is smooth on $\mathbb{R}^{n} \backslash\{0\}$ and satisfies

$$
(1-\varepsilon)\|x\| \leq V(x) \leq(1+\varepsilon) \sigma(\|x\|)
$$

and

$$
\gamma((1+\varepsilon)\|u\|) \leq V(x) \quad \Rightarrow \quad D V(x) \cdot f(x, u) \leq-(1-\varepsilon) g(V(x))
$$

for all $x \in \mathbb{R}^{n} \backslash\{0\}$ and all $u \in U$.

It should be noted that there exists an intermediate object between the discontinuous and the smooth ISDS Lyapunov function, namely a Lipschitz Lyapunov function which satisfies (3.4) in a suitable generalized sense using the theory of viscosity solutions, see [4] for details. While both smooth and Lipschitz Lyapunov functions characterize the optimal gains "in the limit", we conjecture that there are examples in which gains can be exactly characterized by Lipschitz but not by smooth ISDS Lyapunov functions, similar to what was shown recently for $H_{\infty}$ Lyapunov functions in [11].

Theorem 3.2 gives rise to a constructive procedure of computing ISDS robustness gains from Lyapunov functions for the unperturbed system $f(x, 0)$. We illustrate this procedure by three examples.

Example 3.3 Consider a linear system $\dot{x}=f(x, u)=A x+B u$. If we assume ISDS then the matrix $A$ needs to be Hurwitz and we can find a quadratic Lyapunov function $W(x)=x^{T} P x$ for some positive definite matrix $P$ satisfying $c_{1}\|x\|^{2} \leq W(x) \leq c_{2}\|x\|^{2}$ and $D W(x) A x \leq-c_{3}\|x\|^{2}$. Setting $V(x)=\sqrt{W(x) / c_{1}}$ we obtain $\|x\| \leq V(x) \leq c_{4}\|x\|$, $D V(x) A x \leq-c_{5} V(x)$ and $\|D V(x)\| \leq c_{4}$ for $c_{4}=\sqrt{c_{2} / c_{1}}$ and $c_{5}=c_{3} /\left(2 c_{2}\right)$. Fixing some $\lambda \in(0,1)$ we set $\gamma(r)=c_{4}\|B\| r /\left(\lambda c_{5}\right)$. Then we obtain

$$
\gamma(\|u\|) \leq V(x) \quad \Rightarrow \quad D V(x) \cdot f(x, u) \leq-(1-\lambda) c_{5} V(x)=:-g(V(x)) .
$$

Hence $V$ is an ISDS Lyapunov function in the sense of Theorem 3.2 (for each $\varepsilon>0$ ) and we obtain ISDS with $\mu(r, t)=e^{-(1-\lambda) c_{5} t} r, \sigma(r)=c_{4} r$ and $\gamma(r)=c_{4}\|B\| r /\left(\lambda c_{5}\right)$, i.e., exponential convergence and linear overshoot and robustness gains.

This example nicely illustrates the (typical) tradeoff between the attraction rate $\mu$ and the robustness gain $\gamma$, which is represented here by the choice of $\lambda$ : the smaller $\gamma$ becomes the slower convergence can be guaranteed. In the next two examples, showing ISDS estimates for two simple nonlinear systems, we set $\lambda=3 / 4$.

Example 3.4 Consider the system $\dot{x}=f(x, u)=-x+u^{3} / 2$ with $x \in \mathbb{R}, u \in \mathbb{R}$. Using the Lyapunov function $V(x)=|x|$ one obtains $D V(x) f(x, 0)=-|x|=-V(x)$. We choose $\gamma$ such that $\gamma(|u|) \leq V(x)=|x|$ implies $\left|u^{3} / 2\right| \leq 3|x| / 4$, i.e., $\gamma(r)=2 r^{3} / 3$. Then we obtain

$$
\gamma(\|u\|) \leq V(x) \quad \Rightarrow \quad D V(x) \cdot f(x, u) \leq-\frac{1}{4} V(x)=:-g(V(x)),
$$

and consequently ISDS with $\mu(r, t)=e^{-t / 4} r, \sigma(r)=r$ and $\gamma(r)=2 r^{3} / 3$. 
Example 3.5 Consider the system $\dot{x}=f(x, u)=-x^{3}+u$ with $x \in \mathbb{R}, u \in \mathbb{R}$. Again using the Lyapunov function $V(x)=|x|$ one obtains $D V(x) f(x, 0)=-|x|^{3}=-V(x)^{3}$. Here we choose $\gamma$ such that $\gamma(|u|) \leq V(x)=|x|$ implies $|u| \leq 3|x|^{3} / 4$, i.e., $\gamma(r)=\sqrt[3]{4 r / 3}$. Then we obtain

$$
\gamma(\|u\|) \leq V(x) \quad \Rightarrow \quad D V(x) \cdot f(x, u) \leq-\frac{1}{4} V(x)^{3}=:-g(V(x)),
$$

and consequently ISDS with $\mu(r, t)=\sqrt{2 t+4 / r^{2}} /\left(t+2 / r^{2}\right)$ (the solution of $\left.\dot{\mu}=-\mu^{3} / 4\right)$, $\sigma(r)=r$ and $\gamma(r)=\sqrt[3]{4 r / 3}$

\section{Applications}

As a first application, we derive an estimate on a nonlinear stability margin. In [17] it was shown that ISS implies the existence of a stability margin for a perturbed system, however, for ISS it is difficult to derive an estimate for this margin. In contrast to this, the ISDS property easily allows to give an estimate based on the ISDS robustness gain.

Theorem 4.1 Consider a system (2.1) and assume ISDS with $\mu, \sigma$ and $\gamma$ and $U=\mathbb{R}^{m}$, satisfying Assumption 2.3. Consider a Lipschitz map $k: \mathbb{R}^{n} \rightarrow \mathbb{R}_{0}^{+}$satisfying $k(x) \leq$ $\max \left\{\gamma^{-1}(\|x\|), k_{0}\right\}$ for some value $k_{0} \geq 0$. Then for each $x \in \mathbb{R}^{n}$ and all $u \in \mathcal{U}$ with $\|u\|_{\infty} \leq 1$ the trajectories $\varphi_{k}(t, x, u)$ of the system $\dot{x}=f_{k}(x, u):=f(x, k(x) u)$ satisfy

$$
\left\|\varphi_{k}(t, x, u)\right\| \leq \max \left\{\mu(\sigma(\|x\|), t), \gamma\left(k_{0}\right)\right\}
$$

for all $t \geq 0$.

Proof: Fix $\varepsilon>0$ and consider the function $k_{\varepsilon}(x):=(1-\varepsilon) k(x)$. Then for $\varepsilon \rightarrow 0$ the trajectories $\varphi_{\varepsilon}(t, x, u)$ of $\dot{x}=f\left(x, k_{\varepsilon}(x) u\right)$ converge pointwise to $\varphi_{k}(t, x, u)$. Now let $V$ be the ISDS Lyapunov functions from Theorem 3.2 for this $\varepsilon>0$. Then for all $\|u\| \leq 1$ we obtain

$$
D V(x) \cdot f\left(x, k_{\varepsilon}(x) u\right) \leq-(1-\varepsilon) g(V(x))
$$

for all $x \in \mathbb{R}^{n}$ with $V(x) \geq \gamma\left(k_{0}\right)$. Integrating (4.1) we obtain

$$
(1-\varepsilon)\left\|\varphi_{\varepsilon}(t, x, u)\right\| \leq V\left(\varphi_{\varepsilon}(t, x, u)\right) \leq \max \left\{\mu(V(\|x\|),(1-\varepsilon) t), \gamma\left(k_{0}\right)\right\} .
$$

Since all expressions involved are continuous in $\varepsilon$ we obtain the assertion for $\varepsilon \rightarrow 0$.

As a second application we consider the stability of coupled systems. The following theorem is a version of the generalized small gain theorem [7, Theorem 2.1] (in a simplified setting). As for Theorem 4.1, the qualitative result (i.e., asymptotic stability of the coupled system) can be proved using the original ISS property. The advantage of ISDS lies in the estimates for the overshoot and the decay rates of the coupled system.

Theorem 4.2 Consider two systems $\dot{x}_{i}=f\left(x_{i}, u_{i}\right), i=1,2$, of type $(2.1)$ where the $f_{i}$ are Lipschitz in both $x_{i}$ and $u_{i}$. Let $x_{i} \in \mathbb{R}^{n_{i}}, U_{1}=\mathbb{R}^{n_{2}}$ and $U_{2}=\mathbb{R}^{n_{1}}$. Assume that the systems are ISDS with rates $\mu_{i}$ and gains $\sigma_{i}$ and $\gamma_{i}$ and assume that the inequalities $\gamma_{1}\left(\gamma_{2}(r)\right) \leq r$ and $\gamma_{2}\left(\gamma_{1}(r)\right) \leq r$ hold for all $r>0$. Then the coupled system

$$
\dot{x}_{1}=f_{1}\left(x_{1}, x_{2}\right), \quad \dot{x}_{2}=f_{2}\left(x_{2}, x_{1}\right)
$$


is globally asymptotically stable and the trajectories $\left(x_{1}(t), x_{2}(t)\right)$ of $(4.2)$ satisfy

$$
\left\|x_{i}(t)\right\| \leq \delta_{i}\left(\max \left\{\sigma_{i}\left(\left\|x_{i}(0)\right\|\right), \gamma_{i}\left(\sigma_{j}\left(\left\|x_{j}(0)\right\|\right)\right)\right\}, t\right)
$$

for $i=1,2, j=3-i$ and functions $\delta_{i}$ given by

$$
\delta_{i}(r, t):=\sup \left\{\theta_{i}^{t_{1}, s_{1}} \circ \ldots \circ \theta_{i}^{t_{k}, s_{k}}(r) \mid k \geq 1, t_{j}, s_{j} \geq 0, \sum_{j=1}^{k} t_{j}+s_{j}=t\right\}
$$

with $\theta_{1}^{t, s}(r):=\mu_{1}\left(\gamma_{1}\left(\mu_{2}\left(\gamma_{1}^{-1}(r), s\right)\right), t\right)$ and $\theta_{2}^{t, s}(r):=\mu_{2}\left(\gamma_{2}\left(\mu_{1}\left(\gamma_{2}^{-1}(r), s\right)\right), t\right)$. In particular, for all $t \geq 0$ from (4.3) we obtain the overshoot estimates

$$
\left\|x_{i}(t)\right\| \leq \max \left\{\sigma_{i}\left(\left\|x_{i}(0)\right\|\right), \gamma_{i}\left(\sigma_{j}\left(\left\|x_{j}(0)\right\|\right)\right)\right\} .
$$

Proof: One can verify that, defining $\left.\tilde{\mu}_{2}(r, t):=\gamma_{1}\left(\mu_{2}\left(\gamma_{1}^{-1}(r), s\right)\right), t\right), T=\sum_{j=1}^{k} t_{j}$ and $S=\sum_{j=1}^{k} s_{j}$ one has

$$
\delta_{1}(r, t) \leq \min \left\{\mu_{1}(r, T), \tilde{\mu}_{2}(r, S)\right\} \leq \max \left\{\mu_{1}(r, t / 2), \tilde{\mu}_{2}(r, t / 2)\right\},
$$

and analogous for $\delta_{2}$. The last term on the right hand side is a class $\mathcal{K} \mathcal{L}$ function with $\max \left\{\mu_{1}(r, 0), \tilde{\mu}_{2}(r, 0)\right\}=r$, thus the $\delta_{i}$ are bounded from above by class $\mathcal{K} \mathcal{L}$ functions and satisfy $\delta(r, 0)=r$.

Hence we only have to show (4.3), since then global asymptotic stability and the overshoot estimates follow immediately. It is sufficient to show (4.3) for the family of coupled systems

$$
\dot{x}_{1}=f_{1}\left(x_{1}, \eta x_{2}\right), \quad \dot{x}_{2}=f_{2}\left(x_{2}, \eta x_{1}\right)
$$

with $\eta \in(0,1)$, since for the trajectories $x_{i}^{\eta}(t)$ of (4.4) we obtain $\left\|x_{i}^{\eta}(t)\right\| \rightarrow\left\|x_{i}(t)\right\|$ as $\eta \rightarrow 1$, hence if (4.3) holds for $\left\|x_{i}^{\eta}(t)\right\|$ for each $\eta \in(0,1)$, then these estimates also hold for $\left\|x_{i}(t)\right\|$. Thus, we fix $\eta \in(0,1)$ and, to keep the notation simple, again denote the trajectories of (4.4) by $x_{i}(t)$.

Inserting the ISDS estimates for the second system into the ISDS estimate for the first and using the definition of $\delta_{1}$ we obtain

$$
\begin{aligned}
\left\|x_{1}(t)\right\| \leq \max \left\{\delta_{1}\left(\sigma_{1}\left(\left\|x_{1}(0)\right\|\right), t\right), \delta_{1}\left(\gamma_{1}\left(\sigma_{2}\left(\left\|x_{2}(0)\right\|\right)\right), t\right)\right. \\
\left.\max _{\tau \in[0, t]} \mu_{1}\left(\gamma_{1}\left(\eta \max _{s \in[0, \tau]} \mu_{2}\left(\gamma_{2}\left(\eta\left\|x_{1}(s)\right\|\right), \tau-s\right)\right), t-\tau\right)\right\}
\end{aligned}
$$

We investigate the third term of (4.5). For $r \geq 0$ and $t \geq s \geq 0$ we abbreviate $\alpha(t, s, r):=$ $\left.\max _{\tau \in[s, t]} \mu_{1}\left(\gamma_{1}\left(\eta \mu_{2}\left(\gamma_{2}(\eta r), \tau-s\right)\right), t-\tau\right)\right\}$. Since $\gamma_{2}(r) \leq \gamma_{1}^{-1}(r)$ and $\eta<1$ we obtain $\alpha(t, s, r) \leq \delta_{1}(r, t-s)$. Furthermore, $\alpha$ is continuous in all three arguments and satisfies

$$
\left.\max _{s \in[0, t]} \alpha\left(t, s,\left\|x_{1}(s)\right\|\right)=\max _{\tau \in[s, t]} \mu_{1}\left(\gamma_{1}\left(\eta \max _{s \in[0, \tau]} \mu_{2}\left(\gamma_{2}\left(\eta\left\|x_{1}(s)\right\|\right), \tau-s\right)\right), t-\tau\right)\right\} .
$$

Now fix some $t>0$ and consider the inequalities

$$
0<\left\|x_{1}(t)\right\| \leq \max _{s \in[0, t]} \alpha\left(t, s,\left\|x_{1}(s)\right\|\right)
$$


If (4.7) is violated then $\left\|x_{1}(t)\right\|$ must be smaller than one of the first two terms in (4.5) which implies (4.3). Hence assume (4.7).

We define a sequence $t_{k}, k \geq 0$, inductively by choosing $t_{0}=t$ and $t_{k+1} \in\left[0, t_{k}\right]$ such that $\max _{s \in\left[0, t_{k}\right]} \alpha\left(t_{k}, s,\left\|x_{1}(s)\right\|\right)=\alpha\left(t_{k}, t_{k+1},\left\|x_{1}\left(t_{k+1}\right)\right\|\right)$. Then for all $k \geq 0$ we have either

$$
\left\|x_{1}\left(t_{k}\right)\right\|>\max _{s \in\left[0, t_{k}\right]} \alpha\left(t_{k}, s,\left\|x_{1}(s)\right\|\right)
$$

or

$$
\left\|x_{1}\left(t_{k}\right)\right\| \leq \max _{s \in\left[0, t_{k}\right]} \alpha\left(t_{k}, s,\left\|x_{1}(s)\right\|\right) \leq \alpha\left(t_{k}, t_{k+1},\left\|x_{1}\left(t_{k+1}\right)\right\|\right) \leq \delta_{1}\left(\left\|x_{1}\left(t_{k+1}\right)\right\|, t_{k}-t_{k+1}\right) .
$$

Note that (4.9) holds for $k=0$. We claim that there exists $k \geq 1$ such that (4.8) holds. Choosing $k_{0} \geq 1$ minimal with this property we obtain

$$
\left\|x_{1}(t)\right\| \leq \delta_{1}\left(\left\|x_{1}\left(t_{k}\right)\right\|, t-t_{k}\right) \text { for all } k=0, \ldots, k_{0} .
$$

In order to show the existence of this $k_{0}$, observe that the sequence $t_{k}$ is monotone decreasing and bounded from below by 0 , hence it converges to some $t^{*} \geq 0$. If $\left\|x_{1}\left(t^{*}\right)\right\|=0$ then either $k_{0}$ exists (and we are done) or from (4.10) we can conclude $\left\|x_{1}(t)\right\| \leq\left\|x_{1}\left(t_{k}\right)\right\|$ for all $k \geq 0$, thus $\left\|x_{1}(t)\right\|=0$ which contradicts (4.7). Hence $\left\|x_{1}\left(t^{*}\right)\right\|>0$ and from $\alpha(t, t, r) \leq \gamma_{1}\left(\gamma_{2}(\eta r)\right) \leq \eta r<r$ for $r>0$ we obtain $\left\|x_{1}\left(t^{*}\right)\right\|>\alpha\left(t^{*}, t^{*}, x_{1}\left(t^{*}\right)\right)$, thus for $k>0$ sufficiently large we can conclude

$$
\left\|x_{1}\left(t_{k}\right)\right\|>\alpha\left(t_{k}, t_{k+1},\left\|x_{1}\left(t_{k+1}\right)\right\|\right)=\max _{s \in\left[0, t_{k}\right]} \alpha\left(t_{k}, s,\left\|x_{1}(s)\right\|\right)
$$

implying the existence of $k_{0}$.

Since (4.8) holds for $k=k_{0},(4.6)$ and (4.5) imply

$$
\left\|x_{1}\left(t_{k_{0}}\right)\right\| \leq \max \left\{\delta_{1}\left(\sigma_{1}\left(\left\|x_{1}(0)\right\|\right), t_{k_{0}}\right), \delta_{1}\left(\gamma_{1}\left(\sigma_{2}\left(\left\|x_{2}(0)\right\|\right)\right), t_{k_{0}}\right)\right\} .
$$

Combining (4.10) for $k=k_{0}$ and (4.11) yields

$$
\begin{aligned}
\left\|x_{1}(t)\right\| & \leq \delta_{1}\left(\max \left\{\delta_{1}\left(\sigma_{1}\left(\left\|x_{1}(0)\right\|\right), t_{k_{0}}\right), \delta_{1}\left(\gamma_{1}\left(\sigma_{2}\left(\left\|x_{2}(0)\right\|\right)\right), t_{k_{0}}\right)\right\}, t-t_{k_{0}}\right) \\
& =\delta_{1}\left(\max \left\{\sigma_{1}\left(\left\|x_{1}(0)\right\|\right), \gamma_{1}\left(\sigma_{2}\left(\left\|x_{2}(0)\right\|\right)\right)\right\}, t\right),
\end{aligned}
$$

i.e., the desired estimate (4.3) for $i=1$. Since the estimate for $i=2$ follows by symmetry, this shows the claim.

Remark 4.3 A different characterization of the decay rates $\delta_{i}$ in Theorem 4.2 can be obtained if we assume that the gains $\gamma_{i}$ and the class $\mathcal{K} \mathcal{L D}$ functions $\mu_{i}$ satisfy Assumption 2.3 for functions $g_{i}$. In this case, derivating the expressions in the definition of $\delta_{i}(r, t)$, $i=1,2$, with respect to $t$, one sees that the $\delta_{i}$ are bounded from above by the solutions of the one-dimensional differential equations $\dot{r}_{i}=\max \left\{-g_{i}\left(r_{i}\right),-\gamma_{i}^{\prime}\left(\gamma_{i}^{-1}\left(r_{i}\right)\right) g_{j}\left(\gamma_{i}^{-1}\left(r_{i}\right)\right)\right\}$, $r_{i}(r, 0)=r$, where $\gamma_{i}^{\prime}$ denotes the derivative of $\gamma_{i}$ and $j=3-i$.

In the following example we illustrate the quantitative information one can obtain from Theorem 4.2 and Remark 4.3. 
Example 4.4 Consider the two systems from Examples 3.4 and 3.5 with robustness gains $\gamma_{1}(r)=2 r^{3} / 3$ and $\gamma_{2}(r)=\sqrt[3]{4 r / 3}$. Then the coupled system reads $\dot{x}_{1}(t)=-x_{1}(t)+$ $x_{2}(t)^{3} / 2, \dot{x}_{2}(t)=-x_{2}(t)^{3}+x_{1}(t)$. One verifies that the gain condition of Theorem 4.2 is satisfied, hence we can conclude asymptotic stability with overshoot estimates

$$
\left\|x_{1}(t)\right\| \leq \max \left\{\left\|x_{1}(0)\right\|, 2\left\|x_{2}(0)\right\|^{3} / 3\right\}, \quad\left\|x_{2}(t)\right\| \leq \max \left\{\left\|x_{2}(0)\right\|, \sqrt[3]{4\left\|x_{1}(0)\right\| / 3}\right\} .
$$

Using the formula from Remark 4.3 we obtain

$$
\dot{r}_{1}=\max \left\{-c_{1} r_{1},-c_{2} r_{1}^{\frac{5}{3}}\right\}, \quad \dot{r}_{2}=\max \left\{-c_{3} r_{2}^{3},-c_{4} r_{2}\right\}
$$

for suitable constants $c_{1}, \ldots, c_{4}>0$. This shows that far away from the equilibrium exponential convergence can be expected, while in a neighborhood of 0 the rates of convergence in both components will slow down.

\section{Proofs}

The following Lemma will be crucial for all our proofs.

Lemma 5.1 Consider a (possibly discontinuous) function $V: \mathbb{R}^{n} \rightarrow \mathbb{R}_{0}^{+}$. Then the following two statements are equivalent

(i) $V(\varphi(t, x, u)) \leq \max \{\mu(V(x), t), \nu(u, \tau)\}$ for all $t \geq 0$ and all $u \in \mathcal{U}$.

(ii) $V(\varphi(t, x, u)) \leq \mu(a, t)$ for all times $t \geq 0$, all values $a \in \mathbb{R}$ with $a \geq V(x)$ and all $u \in \mathcal{U}$ satisfying $\gamma(\| u(\tau)) \|) \leq \mu(a, \tau)$ for almost all $\tau \in[0, t]$.

Proof: "(i) $\Rightarrow$ (ii)": The definition of $\nu$ immediately implies $\nu(u, t) \leq \mu(a, t)$ for $t, a$ and $u$ satisfying the assumptions from (ii), hence (i) implies (ii).

"(ii) $\Rightarrow$ (i)": Consider an arbitrary $u \in \mathcal{U}$ and $t>0$. We set $a=\max \{V(x), \mu(\nu(u, t),-t)\}$ which implies $\gamma(\| u(\tau)) \|) \leq \mu(a, \tau)$ for almost all $\tau \in[0, t]$. Now either $a=V(x)$ or $\mu(a, t)=\nu(u, t)$ holds. In the first case we obtain $V(\varphi(t, x, u)) \leq \mu(a, t)=\mu(V(x), t)$ while in the second case we have $V(\varphi(t, x, u)) \leq \mu(a, t)=\nu(u, t)$. Thus we can conclude (i).

Now we can turn to the Proof of Theorem 3.1:

"(i) $\Rightarrow$ (ii)" We construct a function for which Lemma 5.1(ii) can be verified. We define

$$
V(x):=\inf \{b \geq 0 \mid\|\varphi(t, x, u)\| \leq \max \{\mu(b, t), \nu(u, t)\} \text { for all } u \in \mathcal{U} \text { and all } t \geq 0\} .
$$

Clearly, the ISDS assumption implies $\|x\| \leq V(x) \leq \sigma(\|x\|)$. It remains to show Lemma 5.1(ii). To this end, fix $x \in \mathbb{R}^{n}, a \geq V(x), t>0$ and $u \in \mathcal{U}$ with $\left.\gamma(\| u(\tau)) \|\right) \leq \mu(a, \tau)$ for almost all $\tau \in[0, t]$. This implies $\nu(u, t+s) \leq \max \{\mu(\mu(a, t), s), \nu(u(t+\cdot), s)\}$ for each $s>0$, thus by the definition of $V$ for any $b>a$ we obtain

$$
\|\varphi(t+s, x, u)\| \leq \max \{\mu(b, t+s), \nu(u, t+s)\} \leq \max \{\mu(\mu(b, t), s), \nu(u(t+\cdot), s)\}
$$

which implies $V(\varphi(t, x, u)) \leq \mu(a, t)$ and thus Lemma 5.1(ii).

"(ii) $\Rightarrow$ (i)" This implication follows immediately using the assumed bounds on $V$.

Throughout the rest of this section we assume Assumption 2.3. For the proof of Theorem 3.2 we need four preliminary lemmata. 
Lemma 5.2 Let $\mu$ be a class $\mathcal{K} \mathcal{L D}$ function, let $\gamma$ be a class $\mathcal{K}_{\infty}$ function and let $x \in \mathbb{R}^{n}$. If a continuous function $V: \mathbb{R}^{n} \rightarrow \mathbb{R}_{0}^{+}$, which is differentiable in $x$, satisfies the inequality

$$
V(\varphi(t, x, u)) \leq \max \{\mu(V(x), t), \nu(u, t)\}
$$

for all $t \geq 0$, all $u \in \mathcal{U}$ and $\nu$ from (2.2), then for all $u \in U$ it satisfies

$$
\gamma(\|u\|)<V(x) \quad \Rightarrow \quad D V(x) \cdot f(x, u) \leq-g(V(x)) .
$$

Proof: Fix $u_{0} \in U$ with $\gamma\left(\left\|u_{0}\right\|\right)<V(x)$ and consider the constant function $u(t) \equiv u_{0}$. By continuity, for all $\tau>0$ small enough we obtain $V(\varphi(\tau, x, u)) \leq \mu(V(x), \tau)$, which implies

$D V(x) \cdot f\left(x, u_{0}\right) \leq \limsup _{\tau \rightarrow 0} \frac{V(\varphi(\tau, x, u))-V(x)}{\tau} \leq \limsup _{\tau \rightarrow 0} \frac{\mu(V(x), \tau)-V(x)}{\tau}=-g(V(x))$,

and thus the claim.

We cannot in general conclude the result for $\gamma(\|u\|)=V(x)$ using continuity in $u$ because $U$ is an arbitrary set which might in particular be discrete. The following Lemma shows that we can nevertheless obtain (5.1) for $\gamma(\|u\|)=V(x)$ if $V$ is continuously differentiable. Furthermore, if $V$ is smooth, then also the converse implication holds.

Lemma 5.3 Let $\mu$ be a class $\mathcal{K} \mathcal{L D}$ function satisfying Assumption 2.3 and let $\gamma$ be a class $\mathcal{K}_{\infty}$ function. Then a continuous function $V: \mathbb{R}^{n} \rightarrow \mathbb{R}_{0}^{+}$which is smooth on $\mathbb{R}^{n} \backslash\{0\}$ satisfies the inequality

$$
V(\varphi(t, x, u)) \leq \max \{\mu(V(x), t), \nu(u, t)\}
$$

for all $x \in \mathbb{R}^{n}, t \geq 0$ and all $u \in \mathcal{U}$, where $\nu$ is given by (2.2), if and only if it satisfies

$$
\gamma(\|u\|) \leq V(x) \quad \Rightarrow \quad D V(x) \cdot f(x, u) \leq-g(V(x))
$$

for all $x \in \mathbb{R}^{n} \backslash\{0\}$ and all $u \in U$.

Proof: "(5.2) $\Rightarrow(5.3)$ ": From (5.1) we already know the desired inequality for $\gamma(\|u\|)<$ $V(x)$. Hence fix $u \in \mathcal{U}$ and $x \in \mathbb{R}^{n} \backslash\{0\}$ with $\gamma(\|u\|)=V(x)$. Since by (5.1) we know $D V(x) \neq 0$ the point $x$ cannot be a local maximum. Hence there exists a sequence of points $x_{i} \rightarrow x$ with $V\left(x_{i}\right)>V(x)=\gamma(\|u\|)$. From (5.1) we obtain $D V\left(x_{i}\right) \cdot f\left(x_{i}, u\right) \leq-g\left(V\left(x_{i}\right)\right)$ for all $i \in \mathbb{N}$, which implies (5.3) by continuity.

"(5.3) $\Rightarrow(5.2) ":$ Fix $x \in \mathbb{R}^{n}$ and $t>0$. Integrating (5.3) we obtain

$$
V(\varphi(t, x, u)) \leq \mu(V(x), t) \text { for all } u \in \mathcal{U} \text { with } \gamma(\|u(\tau)\|) \leq \mu(V(x), t) \text { f.a.a. } \tau \in[0, t],
$$

where $\mu$ solves $\dot{\mu}=-g(\mu), \mu(r, 0)=r$. We claim that (5.4) implies Lemma 5.1(ii).

In order to prove the assertion fix $x \in \mathbb{R}^{n}, a \geq V(x)$ and $t>0$, let $u \in \mathcal{U}$ satisfy $\gamma(\| u(\tau)) \|) \leq \mu(a, \tau)$ for almost all $\tau \in[0, t]$ and assume $V(\varphi(t, x, u))>\mu(a, t)$. Then there exists $\delta>0$ such that $V(\varphi(t, x, u))>\mu(a, t)+\delta$. Now pick an arbitrary $\varepsilon<\delta$ and choose $t^{*}>0$ such that $V\left(\varphi\left(t^{*}, x, u\right)\right)=\mu\left(a, t^{*}\right)+\varepsilon$ and $V(\varphi(\tau, x, u))>\mu(a, \tau)+\varepsilon$ for all $\tau \in\left[t^{*}, t\right]$. From the assumption on $u$ we obtain $\gamma(\|u(\tau)\|) \leq V(\varphi(\tau, x, u))-\varepsilon$ for almost all $\tau \in\left[t^{*}, t\right]$. Using the continuity of $V(\varphi(\tau, x, u))$ in $\tau$ and the Lipschitz property of $g$ 
we can now conclude the existence of times $t_{i}, i=0, \ldots, k$ such that $t_{0}=t^{*}, t_{k}=t$ and $\mu\left(V\left(\varphi\left(t_{i}, x, u\right), t_{i+1}-t_{i}\right) \geq V\left(\varphi\left(t_{i}, x, u\right)\right)-\varepsilon\right.$, which implies $\|u(\tau)\| \leq \mu\left(V\left(\varphi\left(t_{i}, x, u\right)\right)\right.$ for almost all $\tau \in\left[t_{i}, t_{i+1}\right]$. Using (5.4) inductively and applying Gronwall's Lemma we obtain

$$
V(\varphi(t, x, u)) \leq \mu\left(V\left(\varphi\left(t^{*}, x, u\right)\right), t-t^{*}\right) \leq \mu\left(\mu\left(a, t^{*}\right)+\varepsilon, t-t^{*}\right) \leq \mu(a, t)+C \varepsilon
$$

for some suitable $C>0$ which contradicts $V(\varphi(t, x, u))>\mu(a, t)+\delta$ as $\varepsilon \rightarrow 0$ and hence shows Lemma 5.1(ii) and thus the assertion.

The next lemma shows the existence of a Lipschitz ISDS Lyapunov function.

Lemma 5.4 If a system (2.1) is ISDS with rate $\mu$ of class $\mathcal{K} \mathcal{L D}$ satisfying Assumption 2.3 and gains $\sigma$ and $\gamma$ of class $\mathcal{K}_{\infty}$ then for each $\varepsilon>0$ there exists a continuous function $V: \mathbb{R}^{n} \rightarrow \mathbb{R}_{0}^{+}$, which is Lipschitz on $\mathbb{R}^{n} \backslash\{0\}$ and satisfies

$$
\|x\| /(1+\varepsilon) \leq V(x) \leq \sigma(\|x\|)
$$

for all $x \in \mathbb{R}^{n}$ and

$$
\gamma(\|u\|)<V(x) \quad \Rightarrow \quad D V(x) \cdot f(x, u) \leq-(1-\varepsilon) g(V(x))
$$

for almost all $x \in \mathbb{R}^{n}$ and all $u \in U$.

Proof: Fix some $\varepsilon>0$ and set $\rho_{\varepsilon}(r):=\varepsilon\left(1-e^{-r}\right)+1$. Then $\rho_{\varepsilon}$ is strictly increasing for $r>0, \rho_{\varepsilon}(0)=1$ and $\rho_{\varepsilon}(r) \nearrow 1+\varepsilon$ as $r \rightarrow \infty$. Using this function we define

$$
V(x):=\inf \left\{\begin{array}{l|l}
b \geq 0 & \begin{array}{l}
\|\varphi(t, x, u)\| \leq \rho_{\varepsilon}(\mu(b, t)) \max \{\mu(b,(1-\varepsilon) t), \nu(u, t)\} \\
\text { for all } u \in \mathcal{U} \text { and all } t \geq 0
\end{array}
\end{array}\right\} .
$$

Similar to the proof of Theorem 3.1 one verifies (5.5) and (5.6).

We now show the Lipschitz property of $V$. In order to do this pick a compact set $N \subset \mathbb{R}^{n}$ not containing the origin. From the bounds on $V$ we can conclude that there exists a compact interval $I=\left[c_{1}, c_{2}\right] \subset \mathbb{R}^{+}$such that for $x \in N$ the infimum over $b \geq 0$ in (5.7) can be replaced by the infimum over $b \in I$. Now the ISDS property implies the existence of a constant $R>0$ such that $\|\varphi(t, x, u)\| \leq \max \{\mu(R, t), \nu(u, t)\}$ holds for all $x \in N$, all $u \in \mathcal{U}$ and all $t \geq 0$, which implies that we can restrict ourselves to those $u \in \mathcal{U}$ with $\|u\|_{\infty} \leq R$. Furthermore, there exists $T>0$ such that $\mu(R, t)<\mu\left(c_{1},(1-\varepsilon) t\right)$ holds for all $t \geq T$, which implies that we only have to check the inequality for $\|\varphi(t, x, u)\|$ in (5.7) for $t \in[0, T]$. Thus the definition of $V$ eventually reduces to

$$
V(x):=\inf \left\{\begin{array}{l|l}
b \in I & \begin{array}{l}
\|\varphi(t, x, u)\| \leq \rho_{\varepsilon}(\mu(b, t)) \max \{\mu(b,(1-\varepsilon) t), \nu(u, t)\} \\
\text { for all } u \in \mathcal{U} \text { with }\|u\|_{\infty} \leq R \text { and all } t \in[0, T]
\end{array}
\end{array}\right\} .
$$

Now we find constants $L_{1}>0$ and $C_{1}>0$ such that the inequalities $\| \varphi\left(t, x_{1}, u\right)-$ $\varphi\left(t, x_{2}, u\right)\left\|\leq L_{1}\right\| x_{1}-x_{2} \|$ and $\left|\rho_{\varepsilon}\left(\mu\left(a_{1}, t\right)\right)-\rho_{\varepsilon}\left(\mu\left(a_{2}, t\right)\right)\right| \geq C_{1}\left|a_{1}-a_{2}\right|$ hold for all $u \in \mathcal{U}$ with $\|u\|_{\infty} \leq R$, all $t \in[0, T]$, all $a_{1}, a_{2} \in I$ and all $x_{1}, x_{2} \in N$.

We set $L_{N}=L_{1} /\left(C_{1} \mu\left(c_{1}, T\right)\right)$, pick $x_{1}, x_{2} \in N$ and fix $\delta>0$. From (5.8) we can conclude the existence of $b^{*} \in I, t^{*} \in[0, T]$ and $u^{*} \in \mathcal{U}$ with $\|u\|_{\infty} \leq R$ such that $b^{*} \geq V\left(x_{1}\right)-\delta$ and $\left\|\varphi\left(t^{*}, x_{1}, u^{*}\right)\right\|>\rho_{\varepsilon}\left(\mu\left(b^{*}, t^{*}\right)\right) \max \left\{\mu\left(b^{*},(1-\varepsilon) t^{*}\right), \nu\left(u^{*}, t^{*}\right)\right\}$. Then $\left\|\varphi\left(t^{*}, x_{2}, u^{*}\right)\right\| \geq \rho_{\varepsilon}\left(\mu\left(b^{* *}, t^{*}\right)\right) \max \left\{\mu\left(b^{* *},(1-\varepsilon) t^{*}\right), \nu\left(u^{*}, t^{*}\right)\right\}$ holds for all $b^{* *}<b^{*}$ with $\left|b^{* *}-b^{*}\right| \geq L_{N}\left\|x_{1}-x_{2}\right\|$, implying $V\left(x_{2}\right) \geq b^{* *}$ and thus $V\left(x_{1}\right)-V\left(x_{2}\right) \leq L_{N}\left\|x_{1}-x_{2}\right\|+\delta$. 
Since $\delta>0$ was arbitrary and this estimate is symmetric in $x_{1}$ and $x_{2}$ we obtain the desired Lipschitz estimate with constant $L_{N}$.

Finally, since by Rademacher's Theorem (see, e.g., [2, page 216]) a Lipschitz function is differentiable almost everywhere, inequality (5.6) follows from Lemma 5.2.

The following lemma gives a smoothing result for Lipschitz Lyapunov functions.

Lemma 5.5 Consider a continuous function $V: \mathbb{R}^{n} \rightarrow \mathbb{R}_{0}^{+}$, which is Lipschitz on $\mathbb{R}^{n} \backslash\{0\}$ and satisfies

$$
\gamma(\|u\|)<V(x) \quad \Rightarrow \quad D V(x) \cdot f(x, u) \leq-g(V(x))
$$

for almost all $x \in \mathbb{R}^{n}$. Then for each two continuous functions $\alpha_{1}, \alpha_{2}: \mathbb{R}^{n} \backslash\{0\} \rightarrow \mathbb{R}^{+}$ there exists a continuous function $\widetilde{V}: \mathbb{R}^{n} \rightarrow \mathbb{R}_{0}^{+}$, which is smooth on $\mathbb{R}^{n} \backslash\{0\}$ and satisfies

$$
\|V(x)-\widetilde{V}(x)\| \leq \alpha_{1}(x) \quad \text { and } \quad \gamma(\|u\|) \leq V(x) \Rightarrow D \widetilde{V}(x) \cdot f(x, u) \leq-g(\widetilde{V}(x))+\alpha_{2}(x)
$$

for all $x \in \mathbb{R}^{n} \backslash\{0\}$.

Proof: This follows from Theorem B.1 in [9], observing that the proof in [9] (which requires compact $U$ ) remains valid if for any compact subset $K \subset \mathbb{R}^{n}$ we can restrict ourselves to a compact subset of $U$, which is the case here since we only need to consider $\|u\| \leq \gamma^{-1}\left(\max _{x \in K} V(x)\right)$.

Finally, we can turn to the Proof of Theorem 3.2:

Assume ISDS, fix $\varepsilon>0$ and let $\varepsilon_{1}>0$ be such that $1 /\left(1+\varepsilon_{1}\right)^{2} \geq(1-\varepsilon),\left(1+\varepsilon_{1}\right)^{2} \leq(1+\varepsilon)$ and $\left(1-\varepsilon_{1}\right)^{2} \geq(1-\varepsilon)$. Applying Lemma 5.4 with $\varepsilon=\varepsilon_{1}$ we can conclude the existence of a locally Lipschitz (away from 0 ) Lyapunov function $V$ satisfying $\|x\| /\left(1+\varepsilon_{1}\right) \leq V(x) \leq$ $\sigma(\|x\|)$ for all $x \in \mathbb{R}^{n}$ and $\gamma(\|u\|)<V(x) \Rightarrow D V(x) \cdot f(x, u) \leq-\left(1-\varepsilon_{1}\right) g(V(x))$ for almost all $x \in \mathbb{R}^{n}$. Applying Lemma 5.5 with $\alpha_{1}(x)=\min \left\{\gamma\left((1+\varepsilon) \gamma^{-1}(V(x))\right)-V(x), \varepsilon_{1} V(x)\right\}$ and $\alpha_{2}(x)=\varepsilon_{1} g(V(x))$ we obtain a smooth (away from 0) Lyapunov function $\widetilde{V}$ satisfying the desired bounds and, since the choice of $\alpha_{1}$ implies $\gamma((1+\varepsilon)\|u\|) \leq \widetilde{V}(x) \Rightarrow \gamma(\|u\|) \leq$ $V(x)$ we obtain

$$
\gamma((1+\varepsilon)\|u\|) \leq \widetilde{V}(x) \quad \Rightarrow \quad D \widetilde{V}(x) \cdot f(x, u) \leq-\left(1-\varepsilon_{1}\right)^{2} g(\widetilde{V}(x)) \leq-(1-\varepsilon) g(\widetilde{V}(x))
$$

for all $x \in \mathbb{R}^{n} \backslash\{0\}$. Hence $\widetilde{V}$ is the desired Lyapunov function.

Conversely, assume the existence of $V$ for any $\varepsilon>0$ and fix $t>0$. By Lemma 5.3 we obtain $(1-\varepsilon)\|\varphi(t, x, u)\| \leq\left\{\mu((1+\varepsilon) \sigma(\|x\|),(1-\varepsilon) t), \nu_{\varepsilon}(u, t)\right\}$ where $\nu_{\varepsilon}(u, t):=$ $\operatorname{ess} \sup _{\tau \in[0, t]} \mu(\gamma(\|(1+\varepsilon) u(\tau)\|),(1-\varepsilon)(t-\tau))$. Since all these expressions are continuous in $\varepsilon$ we obtain the desired inequality.

\section{References}

[1] P. D. Christofides and A. R. Teel. Singular perturbations and input-to-state stability. IEEE Trans. Autom. Control, 41:1645-1650, 1996.

[2] H. Federer. Geometric Measure Theory. Springer-Verlag, New York, 1969.

[3] L. Grüne. Input-to-state stability of exponentially stabilized semilinear control systems with inhomogenous perturbation. Syst. Control Lett., 38:27-35, 1999. 
[4] L. Grüne. Asymptotic Behavior of Dynamical and Control Systems under Perturbation and Discretization. Lecture Notes in Mathematics. Springer-Verlag, 2002. To appear.

[5] L. Grüne, E. D. Sontag, and F. R. Wirth. Asymptotic stability equals exponential stability, and ISS equals finite energy gain - if you twist your eyes. Syst. Control Lett., 38:127-134, 1999.

[6] A. Isidori. Global almost disturbance decoupling with stability for non minimumphase single-input single-output nonlinear systems. Syst. Control Lett., 28:115-122, 1996.

[7] Z. P. Jiang, A. R. Teel, and L. Praly. Small-gain theorem for ISS systems and applications. Math. Control Signals Syst., 7, 1994.

[8] M. Krstić and H. Deng. Stabilization of Nonlinear Uncertain Systems. Springer-Verlag, London, 1998.

[9] Y. Lin, E. D. Sontag, and Y. Wang. A smooth converse Lyapunov theorem for robust stability. SIAM J. Control Optim., 34:124-160, 1996.

[10] L. Praly and Y. Wang. Stabilization in spite of matched unmodelled dynamics and an equivalent definition of input-to-state stability. Math. of Control, Signals, and Systems, 9:1-33, 1996.

[11] L. Rosier and E. D. Sontag. Remarks regarding the gap between continuous, Lipschitz, and differentiable storage functions for dissipation inequalities. Syst. Control Lett., 41:237-249, 2000 .

[12] R. Sepulchre, M. Jankovic, and P.V. Kokotović. Constructive Nonlinear Control. Springer-Verlag, Berlin, 1997.

[13] E. D. Sontag. Smooth stabilization implies coprime factorization. IEEE Trans. Autom. Control, 34:435-443, 1989.

[14] E. D. Sontag. On the input-to-state stability property. Europ. J. Control, 1:24-36, 1995.

[15] E. D. Sontag. Comments on integral variants of ISS. Syst. Control Lett., 34:93-100, 1998.

[16] E. D. Sontag. The ISS philosophy as a unifying framework for stability-like behavior. In A. Isidori, F. Lamnabhi-Lagarrigue, and W. Respondek, editors, Nonlinear Control in the Year 2000, Volume 2, Lecture Notes in Control and Information Sciences 259, pages 443-468. NCN, Springer Verlag, London, 2000.

[17] E. D. Sontag and Y. Wang. On characterizations of the input-to-state stability property. Syst. Control Lett., 24:351-359, 1995.

[18] J. Tsinias. Input to state stability properties of nonlinear systems and applications to bounded feedback stabilization using saturation. ESAIM Control Optim. Calc. Var., 2:57-85, 1997. 